\title{
RELIABILITY AFTER INSPECTION
}

by John R. Davidson

Langley Research Center

Hampton, Virginia

Presented at the ASTM Symposium on the Fatigue of Composite Materials

Bal Harbour, Florida, December 2-7, 1973.

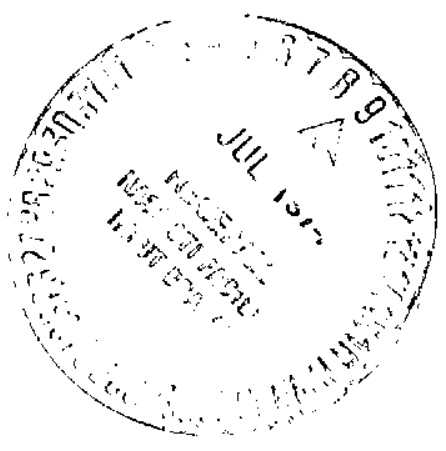

This informal documentation medium is used to provide accelerated or special release of technical information to selected users. The contents may not meet NASA formal editing and publication standards, may be revised, or may be incorporated in another publication.

\section{NATIONAL AERONAUTICS AND SPACE ADMINISTRATION}

LANGLEY RESEARCH CENTER, HAMPTON, VIRGINIA 23665 


\begin{tabular}{|c|c|c|c|c|}
\hline $\begin{array}{l}\text { 1. Report No. } \\
\text { TMX-71969 }\end{array}$ & \multicolumn{2}{|c|}{ 2. Government Accession No. } & \multicolumn{2}{|c|}{ 3. Recipient's Catalog No. } \\
\hline \multicolumn{3}{|l|}{ 4. Title and Subtitle } & \multicolumn{2}{|l|}{ 5. Report Date } \\
\hline \multicolumn{3}{|c|}{ RELIABILITY AFTER INSPECTION } & \multicolumn{2}{|c|}{ 6. Performing Organization Code } \\
\hline 7. Authar(s) & & & \multicolumn{2}{|c|}{ 8. Performing Organization Report No. } \\
\hline John R. Davidson & & & \multicolumn{2}{|l|}{ 10. Work Unit No. } \\
\hline \multicolumn{3}{|c|}{ 9. Performing Organization Name and Address } & & \\
\hline \multicolumn{3}{|c|}{$\begin{array}{l}\text { NASA-Langley Research Center } \\
\text { Hampton, Virginia } 23665\end{array}$} & \multicolumn{2}{|c|}{ 11. Contract or Grant No. } \\
\hline \multicolumn{3}{|c|}{ 12. Sponsoring Agency Name and Address } & \multicolumn{2}{|c|}{ 13. Type of Report and Period Covered } \\
\hline \multicolumn{3}{|c|}{$\begin{array}{l}\text { National Aeronautics and Space Administration } \\
\text { Washington, D.C. } 20546\end{array}$} & \multicolumn{2}{|c|}{ 14. Sponsoring Agency Code } \\
\hline \multicolumn{5}{|l|}{ 15. Supplementary Notes } \\
\hline \multicolumn{5}{|l|}{$\begin{array}{l}\text { 16. Abstract } \\
\text { Finished products a } \\
\text { large that the prod } \\
\text { But flaws are occas } \\
\text { and consequently } \\
\text { the quantit ative rela } \\
\text { defects, the probab } \\
\text { established. Sever } \\
\text { be present; where } \\
\text { the piece; where so } \\
\text { and a general case } \\
\text { others, more or les } \\
\text { inspectable while }\end{array}$} \\
\hline \multicolumn{2}{|c|}{$\begin{array}{l}\text { 17. Key Words (Suggested by Author(s)) (STAR category underlined) } \\
\text { Reliability, non-destructive inspection } \\
\text { Bayes' Theorem, structures, materials, } \\
\text { composites. }\end{array}$} & \multicolumn{3}{|c|}{ 18. Distribution Statement } \\
\hline $\begin{array}{l}\text { 19. Security Classif. (of this report) } \\
\text { Unclassif ied }\end{array}$ & $\begin{array}{l}\text { 20. Security Classif. } \\
\text { Unclas if }\end{array}$ & $\begin{array}{l}\text { this page) } \\
\text { Led }\end{array}$ & $\begin{array}{c}\text { 21. No. of Pages } \\
25\end{array}$ & $\begin{array}{l}\text { 22. Price* } \\
\$ 3.00\end{array}$ \\
\hline
\end{tabular}

The National Technical Information Service, Springfield, Virginia 22151

*Availabte from $\left\{\begin{array}{l}\text { TSTIF/NASA Scientific and Technical information Facility, P.O. Box 33، College Park, MD } 20740 \\ \text { ST }\end{array}\right.$ 


\section{INTRODUCTION}

The chemical and physical processes used to form composite materials and structures are often complex and need careful control if the product is to be of acceptable quality. In a laminate, for example, gas bubbles may cause voids, nonuniform surface preparation may lead to debonded areas between layers, or improper wetting may leave fibers unattached to the matrix. However, finished products are inspected to screen out pieces which contain flaws so large that the product quality is impaired with respect to the intended use. But flaws are occasionally overlooked during nondestructive inspections, and consequently such inspections are not 100 percent reliable.

The purpose of the present paper is to derive the relationships among the probability of having manufacturing defects, the probability of detecting a flaw, and final reliability. Several specific situations are considered. First, equations for the simple situation where only one flaw can be present is used to introduce the relationships in a Bayes' Theorem approach to the assessment of the final reliability. Next, situations which are prevelant in composites manufacturing are considered. These include a case where flaws may occur randomly on a laminate surface or throughout a volume, where only the mean number of flaws is known and where the actual number of flaws in a given piece is a Poisson distributed random number which varies about this mean. This solution is then expanded to include the more general instance where some area or volume may not be amenable to inspection and must go uninspected. 
SYMBOLS
A
A flaw exists
$A^{\prime}$.
A flaw does not exist
B
A flaw is indicated
$B^{\prime}$
A flaw is not indicated
b
Number of subareas
$\mathrm{L}$
Length which may contain flaws, m
n
Number of subareas with flaws
$P_{1}$
Probability when flaw site is known
$\mathrm{P}_{2}$
Probability when flaw site unknown; many flaw'
total inspection
$\mathbf{P}_{3}$
Probability when flaw site unknown; many $f$ aws;
partial inspection
$P_{4}$
Probability when flaw site unknown; many flaws;
partial inspection; flaw rate in uninspected region differs from rate in inspected region
$\mathrm{P}_{5}$
Probability when flaw site unknown; many flaws;
Probability of event A occurring
$P[A B]$
Probability that both $A$ and $B$ occur
$\mathbf{P}[\mathrm{A} \mid \mathrm{B}]$
Probability of event A occurring, given that event $B$
$p(n)$ has occurred
Probability that $n$ flaws exist 
R

$R_{1}, R_{2}, R_{3}, R_{4}, R_{5}$

$\mathrm{S}$

V

$\lambda$

$\rho$

Subscripts

a

b
Reliability after inspection

Reliabilities; subscripts denote cases 1ike those for subscripted probabilities

Area which may contain flaws, $\mathrm{m}^{2}$

Volume which may contain flaws, $\mathrm{m}^{3}$

Mean number of flaws per unit length, area or volume;

$$
\mathrm{m}^{-1}, \mathrm{~m}^{-2}, \mathrm{~m}^{-3}
$$

Proportion of length, area, or volume of piece inspected
Refers to parameters associated with the uninspectable portion of the structure

Refers to parameters associated with the inspectable portion of the structure 
Quantification of the Inspection Procedures

Perhaps no inspection procedure can be 100 percent certain to find all flaws. To be useful in reliability calculations the amount of certainty (or uncertainty) must be quantified, and to be quantified the inspection procedure itself must be tested.

One way to test the inspection procedure is to inspect pieces in which flaws are known to exist. Of course, the inspectors themselves should be ignorant about the introduced flaws if the test is to yield a fair measure of the inspection procedures.

If a maximum permissible flaw size is stated, then a "failure" can be defined as the failure to detect any flaw larger than the permitted maximum. A "success" occurs whenever such larger flaws are discovered. The probability of detection during inspection can be calculated from the relative number of successes and failures. The probability is stated by two terms -- probability and confidence [1]. For example, if a total of 50 flawed parts are inspected, and 45 flaws are detected, one has 95 percent confidence that the future proportion of flaws detected will be

$$
.78 \leq \mathrm{p} \leq .97
$$

This proportion is taken as the probability of detecting a flawed part. To be conservative, the above data would establish the working probability at .78. The interval can, of course, be narrowed by testing more specimens or by finding a larger proportion of flaws -- that is to say, having a more effective procedure. 


\section{ANALYSIS}

\section{Flaw Site Known}

The simplest situation occurs when the location of, the potential flaw is known. The flaw, of course, may or may not exist. The problem is to decide whether or not a $\mathrm{flaw}$ is present at the specified location. A11 of those pieces in which flaws are indicated will be rejected or repaired. A11 of those for which no flaws are indicated will be passed. The reliability of the final product is the ratio of flawless pieces passed to total pieces passed, which is also the probability that no flaws exist, given that no flaws were indicated:

$$
R_{1}=P\left[A^{\prime} \mid B^{\prime}\right]
$$

An equation for $R_{1}$ is derived in [2]; the derivation is sketched in Appendix A.V The result is

$$
R_{1}=P_{1}\left[A^{\prime} \mid B^{\prime}\right]=\frac{1}{1+\frac{\left(1-P_{1}[B \mid A]\right) P_{1}[A]}{\left(1-P_{1}[A]\right) P_{1}\left[B^{\prime} \mid A^{\prime}\right]}}
$$

In equation (2), $\mathrm{R}_{1}$ is expressed in terms of known quantities: the probability of detecting an existing $f 1 a w, P[B \mid A]$; the probability that no flaws are indicated if they are not there, $P\left[B^{\prime} / A^{\prime}\right]$; and the unreliability before inspection or probability of a flaw existing before inspection, $P[A]$. 
Equation (2) is a basic equation resulting from Bayes' Theorem. It is one of the simplest. It is sulted for flaw detection when $P[B \mid A]$ is defined, for example, as the probability of detecting all flaws larger than a specified critical size. For an analysis where $P[B \mid A]$ varies with flaw size, or where flaw growth is important, see [3].

Flaw Site Unknown; 100 Percent of the Line, Area, or Volume Inspected

Distribution function for the number of flaws.-In a great many practica1 situations more than one flaw might possibly exist in a part. The distribution function for the number of flaws gives the probability that any specific number of flaws might exist in a part. Frequently, a flaw might be regarded as a "rare event" in the sense that most of the part length, area, or volume is unflawed. One of the first steps, then, is to establish a probability distribution for the total number of flaws in a part.

For the "rare event" situation, and where a flaw is equally likely to be anywhere throughout the area, the probability distribution function for the number of flaws in a part is the Poisson distribution (see Appendix B)

$$
p(n)=\frac{(\lambda s)^{n} e^{-\lambda s}}{n !}
$$

where $p(n)$ is the probability that the part contains $n$ flaws. For bond lines or line welds, $\lambda$ is the mean number of flaws per unit length, and $S$ the length; for laminates $\lambda$ is the mean number of flaws per unit area, 
and $S$ is the area; for large solids, $\lambda$ is the mean number of flaws per unit volume, and $s$ is the volume of the part. Whereas $n$ is, of course, an integer, $\lambda$ may be a decimal. The product, $\lambda s$, obviously is the mean number of flaws per part; the product need not be an integer.

Reliability when flaws axe distributed. -When several flaws are distributed over a surface, only one flaw need be detected for the piece to be rejected. If the detection of any flaw is assumed to be independent of any other existing flaw

$$
P\left[A B^{\prime}\right]=\sum_{n=1}^{\infty} P\left[A_{n}\right] P\left[B^{\prime} \mid A_{n}\right]
$$

where $P\left[A_{n}\right]$ is the probability that exactly $n$ flaws are present over $S$, and $P\left[B^{\prime} \mid A_{n}\right]$ is the probability that all of them escape detection. To get the total probability that a flawed niece passes inspection, one must sum over all possible number of flaws, $\mathbf{n}$.

obviously, under the assumptions of the previous subsection

$$
P\left[A_{n}\right]=P(n)=\frac{(\lambda S)^{n}}{n !} e^{-\lambda S}
$$

Also, under the assumptions of flaw independence and detection independence, the rules of conditional probability hold, and

$$
P\left[B^{\prime} \mid A_{n}\right]=\left(P\left[B^{\prime} \mid A_{1}\right]\right)^{n}=\left(P\left[B^{\prime} \mid A\right]\right)^{n}
$$


Consequently, equation (4) becomes

$$
P\left[A B^{\prime}\right]=e^{-\lambda S} \sum_{n=1}^{\infty} \frac{(\lambda S)^{n}}{n !}\left(P\left[B^{\prime} \mid A\right]\right)^{n}
$$

The remaining derivation is somewhat like that for $R_{1}$ (see Appendix $C$ )

$$
R_{2}=\frac{1}{1+\frac{\exp \left\{\lambda S P\left[B^{\prime} \mid A\right]\right\}-1}{P\left[B^{\prime} \mid A^{\prime}\right]}}
$$

which is analogous to equation (2).

Flaw Site Unknown;

Only Part of the Line, Area, or Volume is Inspectable

In many practical situations, portions of a structure are inaccessible for inspection. The portions may contain flaws. Because uninspectable areas may be conservatively constructed to compensate for uninspectability, the expected number of critical flaws per unit area within them may differ from the number within inspectable areas. In this section the equations for reliability when portions of the structure are uninspectable will be derived. The general case is where

$$
\lambda_{\mathrm{a}} \neq \lambda_{\mathrm{b}}
$$

that is to say, when the flaw rate of occurrence in the inspectable region differs from the rate in the uninspectable region. 
The desired equation is easily derived from equations (3) and (8). The probability that no flaws exist in the uninspected region is

$$
p(0)=\exp \left\{-(1-\rho) \lambda_{a} s\right\}
$$

where $(1-\rho)$ is the proportion of $S$ which is not inspected. The probability that no flaw exists in the inspected region after inspection is

$$
\mathrm{R}_{2}=\frac{1}{1+\frac{\exp \left\{\rho \lambda_{\mathrm{b}^{S}} \mathrm{P}\left[\mathrm{B}^{\top} \mid \mathrm{A}\right]\right\}-1}{\mathrm{P}\left[\mathrm{B}^{\prime} / \mathrm{A}^{\prime}\right]}}
$$

As long as the probability of occurrence of flaws in one region is independent from that in the other, the law of compound probability applies, so that

$$
\mathrm{R}_{4}=\mathrm{p}(0) \mathrm{R}_{2}=\frac{\exp \left\{-(1-\rho) \lambda_{\mathrm{a}} \mathrm{S}\right\}}{1+\frac{\left.\exp \left\{\rho \lambda_{\mathrm{bSP}}\left[\mathrm{B}^{\prime}\right] \mathrm{A}\right]\right\}-1}{\mathrm{P}\left[\mathrm{B}^{\prime} \mid \mathrm{A}^{\prime}\right]}}
$$

Case where $\lambda_{a}=\lambda_{b}$. -If the flaw density is the same in the uninspected region as in the inspected region

$$
\lambda_{\mathrm{a}}=\lambda_{\mathrm{b}}=\lambda
$$

and

$$
\mathrm{R}_{3}=\frac{\exp \{-(1-\rho) \lambda S\}}{1+\frac{\left.\exp \left\{\rho \lambda \mathrm{SP}\left[\mathrm{B}^{\prime}\right] \mathrm{A}\right]\right\}-1}{\mathrm{P}\left[\mathrm{B}^{\prime} \mid \mathrm{A}^{\prime}\right]}}
$$


Many sections.--If a structure contains many sections with various flaw densities, the rules of compound probability can be applied,in the same straightforward fashion as they were in deriving equation (11). For $m$ uninspectable regions and $l$ inspectable regions the general form of the equation is

$$
R_{5}=\frac{\exp \left(-\sum_{k=1}^{m} \lambda_{k} s_{k}\right)}{\prod_{j=1}^{l}\left\{1+\frac{\exp \left[\lambda_{j} S_{j} P_{j}\left[B^{\prime} \mid A\right]\right]-1}{P_{j}\left[B^{\prime} \mid A^{\prime}\right]}\right\}}
$$

where $\mathrm{P}_{j}[\quad]$ is used to indicate that the probabilities of detection or nondetection might vary among regions. 


\section{RESULTS AND DISCUSSION}

The dependence of reliability after inspection upon the reliability before inspection, the probability of detecting an existing flaw, the number of flaws, and the fraction of area inspectable can be illustrated by considering $R_{1}, R_{2}$, and $R_{3}$. Reliabilities $R_{4}$ and $R_{5}$ are simply compounded from the first three.

Figure 1 shows how $R_{1}$ varies with the reliability before inspection and the probability of detecting an existing flaw. If a highly reliable end-product is desired -- for example, with $R_{1}=.99$ or greater -- the figure shows that the reliability before inspection must be at least.9 when the probability of detection is .9 , or that some way must be found to raise the probability of detection above.9. In general, if the probability of flaw detection is at least .9 and the reliability before inspection is at least .9 , then the unreliability after inspection will always be almost an order of magnitude less than the unreliability before inspection.

When flaws are distributed over the surface of a molding or a laminate, the final reliability depends upon the flaw density and the total area. But this dependence can be discussed in terms of the mean number of total flaws, $\lambda s$, as a single parameter. When high reliabilities $\left(R_{2} \geq .95\right)$ are desired, figure 2 shows that the probability of flaw detection, $P[B \mid A]$, must be very high (.95 or greater) if the mean number of flaws is one or greater. To a large part, $R_{2}$ depends strongly upon detection probability when more than one flaw is probable. Again, high reliabilities after inspection are associated with components that had high reliability before the inspection $\left(\lambda S<10^{-1}\right)$. 
Figure 3 illustrates the effect of partial inspection. If only 75 percent of the surface can be inspected, high reliabilities after inspection can only be obtained when reliabilities were high before inspection, regardless of how certain the nondestructive inspection method is to find a crack in the inspectable portion. This situation happens because the uninspected region has a generally unacceptable probability of containing a flaw if $\lambda \mathrm{S} \geq 1$. 


\section{CONCLUDING REMARKS}

Equations were derived for the reliability of a composite material part after it was inspected. The equations related the reliability after inspection to the probability of detecting an existing flaw and the reliability before inspection. Equations were developed for many practical cases: where the potential flaw site was known; where a random number of flaws were distributed randomly over an area (or line, or throughout a volume); where only a fraction of the total area (length or volume) could be inspected; where varfous subsections were more likely to contain flaws than others; and where some of these subsections may require nondestructive inspection techniques that have different probabilities of detecting existing flaws.

The numerical examples showed that an inspection procedure with a .9 probability of detecting an existing flaw can reduce unreliability by almost an order of magnitude if the part reliability is about . 9 or higher before inspection. The examples also showed that a not very reliable laminate should be inspected over one-hundred percent of its surface if the final reliability is to be high.

The one consistent point brought out by the numerical calculations was that the surest way to have a reliable component after inspection was to start with a reliable component before inspection. 
APPENDIX A

The probability that no flaws exist, given that no flaws were indicated during a nondestructive inspection must be expressed in terms of known quantities. The derivation borrows directly from the concepts of probability theory. For example, the probability that $A^{\prime}$ and $B^{\prime}$ occur is

$$
\mathrm{P}_{1}\left[\mathrm{~A}^{\prime} \mathrm{B}^{\prime}\right]=\mathrm{P}_{1}\left[\mathrm{~A}^{\prime} \mid \mathrm{B}^{\prime}\right] \mathrm{P}_{1}\left[\mathrm{~B}^{\prime}\right]
$$

from which

$$
P_{1}\left[A^{\prime} \mid B^{\prime}\right]=\frac{P_{1}\left[A^{\prime} B^{\prime}\right]}{P_{1}\left[B^{\prime}\right]}
$$

But, since $B^{\prime}$ can occur if either $A$ or $A^{\prime}$ occurs,

$$
\mathrm{P}_{1}\left[\mathrm{~B}^{\prime}\right]=\mathrm{P}_{1}\left[\mathrm{~B}^{\prime} \mid \mathrm{A}\right] \mathrm{P}_{1}[\mathrm{~A}]+\mathrm{P}_{1}\left[\mathrm{~B}^{\prime} \mid \mathrm{A}^{\prime}\right] \mathrm{P}_{1}\left[\mathrm{~A}^{\prime}\right]
$$

Also, by analogy with equations (Al)

$$
P_{1}\left[A^{\prime} B^{\prime}\right]=P_{1}\left[B^{\prime} \mid A^{\prime}\right] P_{1}\left[A^{\prime}\right]
$$


If equations (A3) and (A4) are substituted into equation (A2) the result is

$$
R_{1}=P_{1}\left[A^{\prime} \mid B^{\prime}\right]=\frac{1}{1+\frac{P_{1}\left[B^{\prime} \mid A\right] P_{1}[A]}{P_{1}\left[B^{\prime} \mid A^{\prime}\right] P_{1}\left[A^{\prime}\right]}}
$$

But, because some events are mutually exclusive,

$$
P_{1}\left[A^{\prime}\right]=1-P_{1}[A]
$$

and

$$
P_{1}\left[B^{\prime} \mid A\right]=1-P_{1}[B \mid A]
$$

Consequently, equation (A5) can be expressed in terms of the unreliability before inspection, $\mathrm{P}_{1}[\mathrm{~A}]$, and the probability of detecting a flawed part, $\mathrm{P}_{1}[\mathrm{~B} \mid \mathrm{A}]$

$$
\mathrm{R}_{1}=\frac{1}{1+\frac{\left(1-\mathrm{P}_{1}[\mathrm{~B} \mid \mathrm{A}]\right) \mathrm{P}_{1}[\mathrm{~A}]}{\mathrm{P}_{1}\left[\mathrm{~B}^{\prime} \mid \mathrm{A}^{\prime}\right]\left(1-\mathrm{P}_{1}[\mathrm{~A}]\right)}}
$$




\section{APPENDIX B}

The equation for the mass density function for the number of flaws over an area will be derived. The result can be easily converted to apply to line problems or volume problems, as is discussed in the body of the paper.

First, the number of flaws on a surface must be determined. The number of flaws over a surface $S$ will be assumed to be a random number; consequently an area under inspection might have $0,1,2, \ldots$ or any number $n$ flaws.

Suppose, for a moment, that the area $S$ is subdivided into $b$ equal small areas, and that the probability that an unacceptably large flaw is present in a specified subarea is proportional to the area of the subarea. Then, if $\lambda$ is the constant of proportionality

$$
p_{1}=\lambda\left(\frac{s}{b}\right)
$$

The probability of no flaws in $(\mathrm{S} / \mathrm{b})$ is

$$
\mathbf{p}_{0}=1-\frac{\lambda s}{b}-\operatorname{Order}\left(\frac{\lambda s}{b}\right)^{2}
$$

where the third term is the probability that two or more flaws exist in the subarea; this term is of higher order than the second. If $b$ is chosen to be large enough, the third term becomes negligible. 
The probability of finding $n$ subareas with flaws is a series of Bernoulli trials, where each subarea $\mathrm{S} / \mathrm{b}$ is a trial with probability of success $p_{1}$, and failure po [2].

$$
\begin{aligned}
p(n ; b) & =\frac{b !}{(b-n) ! n !}\left(p_{1}\right)^{n}\left(p_{0}\right)^{b-n} \\
& =\frac{b !}{(b-n) ! n !} \frac{\lambda S}{b} n\left(1-\frac{\lambda s}{b}\right)^{b-n}
\end{aligned}
$$

But the subdivision into subareas was arbitrary, and in no way can an arbitrary subdivision alter the mean number of unacceptable flaws ( $\lambda S$ ) contained in $S$. Therefore,

$$
p(n)=\lim _{\substack{b \rightarrow \infty \\ \lambda S=\text { constant }}} p(n ; b)=\frac{(\lambda S)^{n} e^{-\lambda S}}{n !}
$$

Equation (B4) gives the probability that exactly $n$ flaws are present over the area $S$. From the derivation, $\lambda$ can be seen to be the average number of flaws per unit area. Whereas $\lambda$ and $\lambda S$ need not be integers, $n$ obviously must be an integer. Equation (B4) is the Poisson distribution, and is tabulated in most elementary statistics texts. 


\section{APPENDIX C}

Equation (7) can be related to equation (8) through some of the relationships of elementary probability theory. As in Appendix A

$$
P\left[A B^{\prime}\right]=P\left[A \mid B^{\prime}\right] P\left[B^{\prime}\right]=P\left[B^{\prime} \mid A\right] P[A]
$$

The "unreliability" is the probability of accepting a flawed part, and, parallel to equation (A2) is

$$
1-R_{2}=P\left[A \mid B^{\prime}\right]=\frac{P\left[A B^{\prime}\right]}{P\left[B^{\prime}\right]}
$$

Also

$$
\begin{aligned}
P\left[B^{\prime}\right] & =P\left[A B^{\prime}\right]+P\left[A^{\prime} B^{\prime}\right] \\
& =P\left[A B^{\prime}\right]+P\left[B^{\prime} \mid A^{\prime}\right] P\left[A^{\prime}\right]
\end{aligned}
$$

Equations (B2), (B3), and (A3) can be combined to yield

$$
\mathrm{R}_{2}=1-\frac{1}{1+\frac{\left.\mathrm{P}\left[\mathrm{B}^{\prime}\right] \mathrm{A}\right] \mathrm{P}\left[\mathrm{A}^{\prime}\right]}{\mathrm{P}\left[\mathrm{AB} \mathrm{B}^{\prime}\right]}}
$$


Using equation (7) in equation (B4), and noting that

$$
P\left[A^{\prime}\right]=p(0)=e^{-\lambda S}
$$

and that

$$
\sum_{n=1}^{\infty} \frac{x^{n}}{n !}=e^{x}-1
$$

equation (B4) becomes

$$
\mathrm{R}_{2}=\frac{1}{1+\frac{\exp \left\{\lambda S \frac{P}{\mathrm{P}}\left[\mathrm{B}^{\prime} \mid \mathrm{A}\right]\right\}-1}{\mathrm{P}\left[\mathrm{B}^{\dagger} \mid \mathrm{A}^{\prime}\right]}}
$$




\section{REFERENCES}

[1] Dixon, Wilford J.; and Massey, Frank J., Jr.: Introduction to Statistical Analysis. Second Edition, McGraw Hill Book Co., Inc., New York 1957.

[2] Parzen, Emmanuel: Modern Probability Theory and Its Applications. John Wiley \& Sons, Inc., New York, 1962. Library of Congress 60-6456.

[3] Davidson, John R.: Reliability and Structural Integrity. Presented at the 10th Anniversary Meeting of the Society of Engineering Science, Raleigh, NC, Nov. 1973. NASA TM X-71934. 


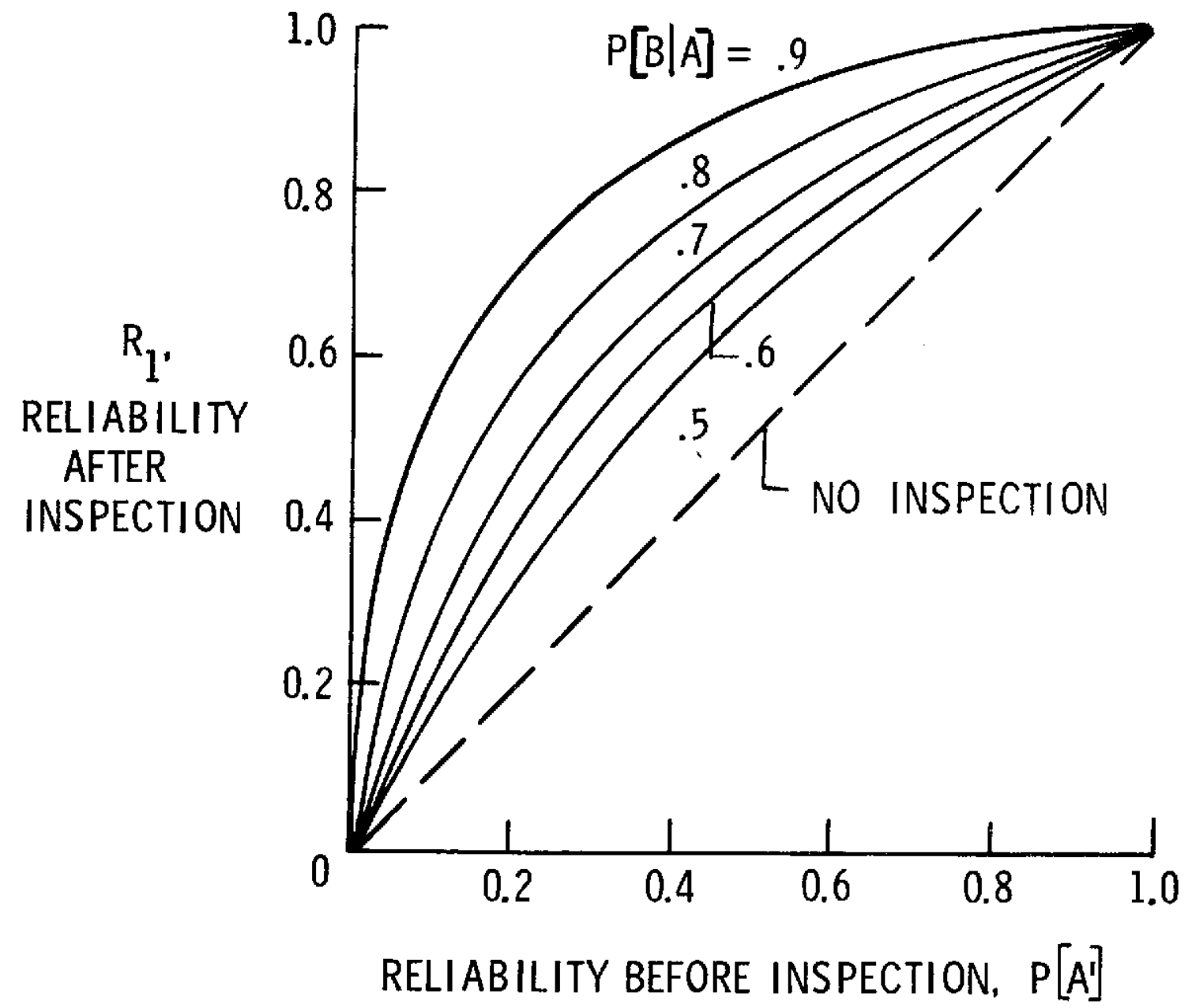

Figure 1.-Variation of reliability after inspection as a function of reliability before inspection. The probability of identifying an existing flaw is a parameter. The false alarm rate, $\mathrm{P}\left[\mathrm{B} \mid \mathrm{A}^{\prime}\right]=.02$. 


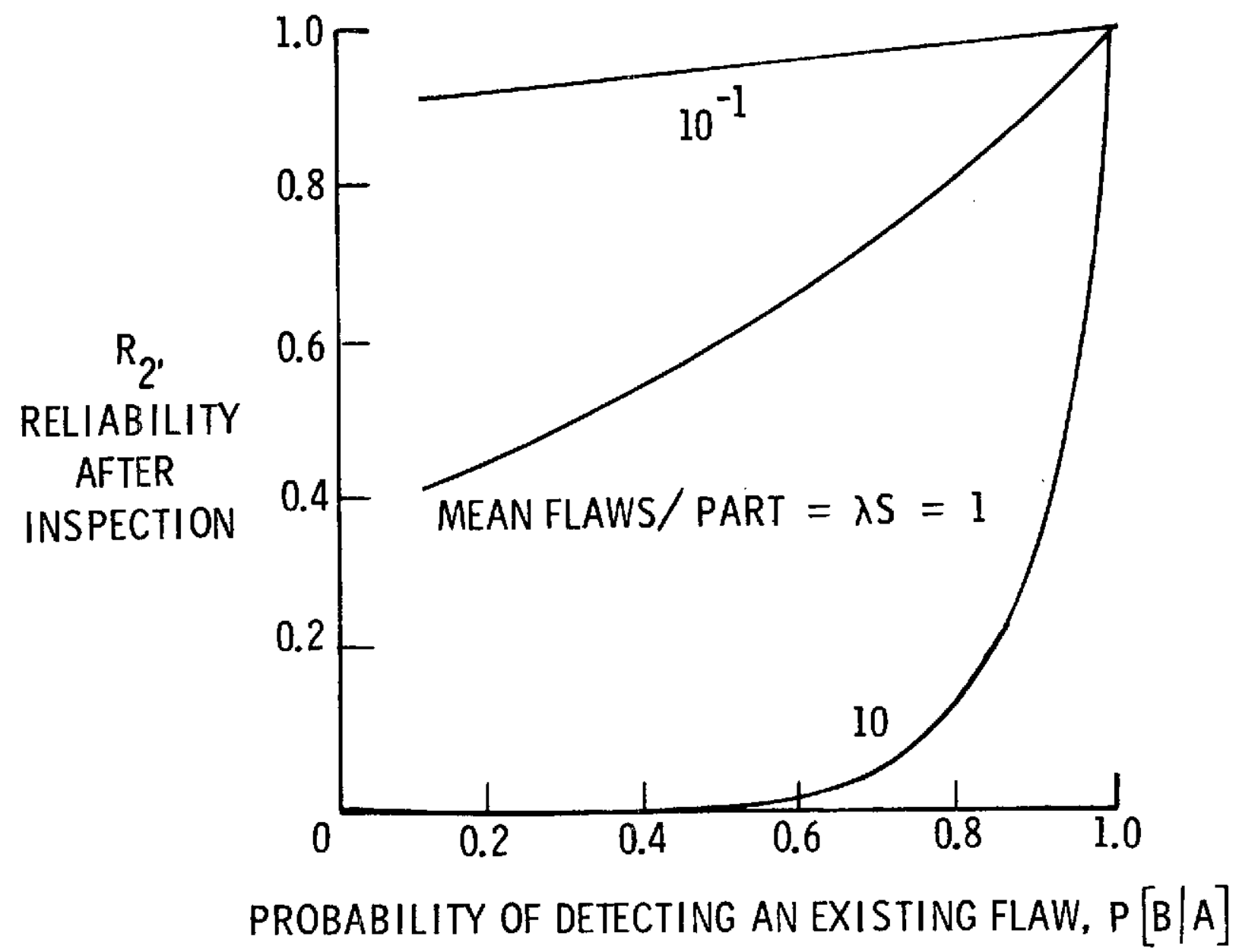

Figure 2.-Variation of reliability as a function of the probability of detecting an existing flaw; the mean number of existing flaws is the parameter. False alarm rate, $P\left[B \mid A^{\prime}\right]=.02$. 


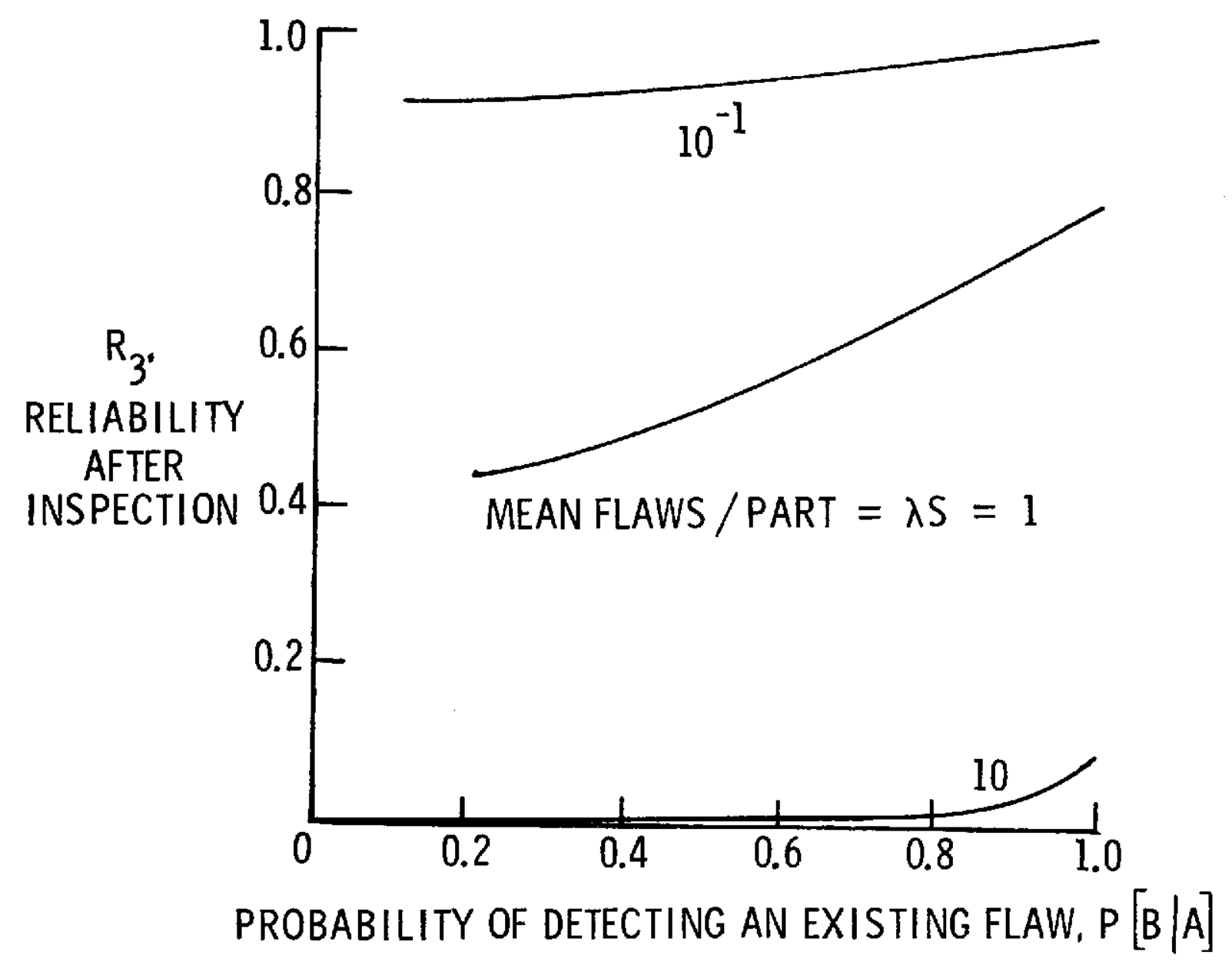

Figure 3.-Variation of reliability as a function of the probability of detecting an existing flaw when only $75 \%$ of the total area inspected; the mean number of existing alarm rate $P\left[B \mid A^{\prime}\right]=.02$. 